\title{
Twisting and untwisting of twisted nematic elastomers
}

\author{
Zoey S. Davidson $\odot,{ }^{1, *}$ Nadia Kapernaum, ${ }^{2}$ Jonathan Fiene $\odot,{ }^{3}$ Frank Giesselmann, ${ }^{2}$ and Metin Sitti $\odot^{1}$ \\ ${ }^{1}$ Physical Intelligence Department, Max Planck Institute for Intelligent Systems, Heisenbergstrasse 3, 70569 Stuttgart, Germany \\ ${ }^{2}$ Institute of Physical Chemistry, University of Stuttgart, Pfaffenwaldring 55, 70569 Stuttgart, Germany \\ ${ }^{3}$ Robotics Central Scientific Facility, Max Planck Institute for Intelligent Systems, Heisenbergstrasse 3, 70569 Stuttgart, Germany
}

(Received 28 April 2020; accepted 9 July 2020; published 9 October 2020)

\begin{abstract}
A mechanical stretch applied to a monodomain twisted nematic elastomer thin film untwists the helical director distribution into a uniaxially aligned state. If the film thickness is less than the helical pitch, untwisting behavior is stretching direction dependent in the plane normal to the helix axis. We investigate untwisting of twisted nematic elastomers by optical, mechanical, and x-ray scattering methods. We adapt a model for cholesteric elastomers to our observations and find that the material exhibits characteristics with the combined properties of both a smectic and twisted nematic configuration.
\end{abstract}

DOI: 10.1103/PhysRevMaterials.4.105601

Advances in chemistry and fabrication methods have greatly expanded the possibilities for technologies utilizing liquid crystal elastomers (LCEs) as well as studying fundamental properties of LCE materials. In particular, clickchemistry [1-3] and photoalignment [4,5] methods have enabled the facile synthesis of LCEs with giant elastic anisotropy and complex molecular programming, respectively. One arrangement of LCEs that has drawn particular attention is that of a cholesteric liquid crystal elastomer [6]. In a cholesteric LCE, the nematic director twists about a helix axis with a material intrinsic pitch $p$. This pitch imparts important optical functionality to the material and is the basis of many optical applications [7-9]. When $p$ is the same wavelength as an incident light beam, the cholesteric LCE will selectively reflect the light of the matching wavelength. When $p$ is much greater than the wavelength of incident light, the so-called Mauguin regime, the cholesteric LCE director guides the light polarization. In the case where $p$ is set by the boundary conditions (and thus necessarily greater than twice the LCE sample thickness), the system is referred to as a twisted nematic LCE.

The twisted nematic regime imparts interesting mechanical behavior to the bulk sample. A uniaxially aligned LCE film has two principal bulk moduli: one along the director and one in the plane normal to the director. A short-pitch cholesteric LCE has a principal modulus along its helix axis and is isotropic in the plane transverse to the helix $[10,11]$. However, in a film of twisted nematic LCE, again, there is one principal bulk modulus along the helix axis, but perpendicular to the helix axis, the modulus depends on the portion of the helix

\footnotetext{
*davidson@is.mpg.de
}

Published by the American Physical Society under the terms of the Creative Commons Attribution 4.0 International license. Further distribution of this work must maintain attribution to the author(s) and the published article's title, journal citation, and DOI. Open access publication funded by the Max Planck Society. phase present. Crucially, the stretching behavior will exhibit a critical phenomenon only if the portion of the helix phase present contains a director perpendicular to the stretching direction. In other words, for portions of the helix phase with a director component perpendicular to the stretching direction, there will be a critical stretch where the perpendicular director component must select an untwisting direction. This effect has previously been called director pinning in cholesteric LCEs [10]. For long-pitch twisted nematic LCEs, the film geometry and portion of the helix phase present will also affect the thermal actuation characteristics [12].

Smectic elastomers exhibit much larger elastic anisotropy than nematic or cholesteric elastomers due to the constant layer spacing between LCE molecules [13]. Typically, smectic layering is incompatible with a twisted director structure unless a highly defected twist-grain boundary structure is present [14]. However, localized short-range smectic ordering enables both twisted nematiclike and smecticlike behaviors $[15,16]$. The smectic clusters which exist in equilibrium in the precursor LCE melt are "locked in" by the photo crosslinking of the LCE. We investigated the interplay of the twisted director field and short-range smectic ordering in the properties of LCE films. We found the material exhibits properties consistent with both twisted nematic and smectic- $C$ phases, which results in mechanical properties that are distinct from either phase on its own.

We adapted a model for short-pitch cholesteric LCEs developed by Warner et al. [10,11] to a long-pitch twisted nematic LCE, which we also fabricated. Following the same notation as in [10], the twisted nematic LCE helix has initial wave number $q_{0}=\pi / p$, where $p$ is at least four times the film thickness. As we stretch the twisted nematic LCE $\lambda$, there is a volume-conserving contraction, both along the helix $\lambda_{z z}$ and in the direction normal to both the helix and the stretch $\lambda_{j j}$. A key feature of the Warner model is its prediction of the director angle $\phi(z ; \lambda)$ along the helix axis as the film is stretched $\lambda$. In our modification of this model, we restricted the limits of $z$, such that the total twist $q_{0} z$ is less than $90^{\circ}$ (typically, $80^{\circ}$ in experiments). We additionally considered a 

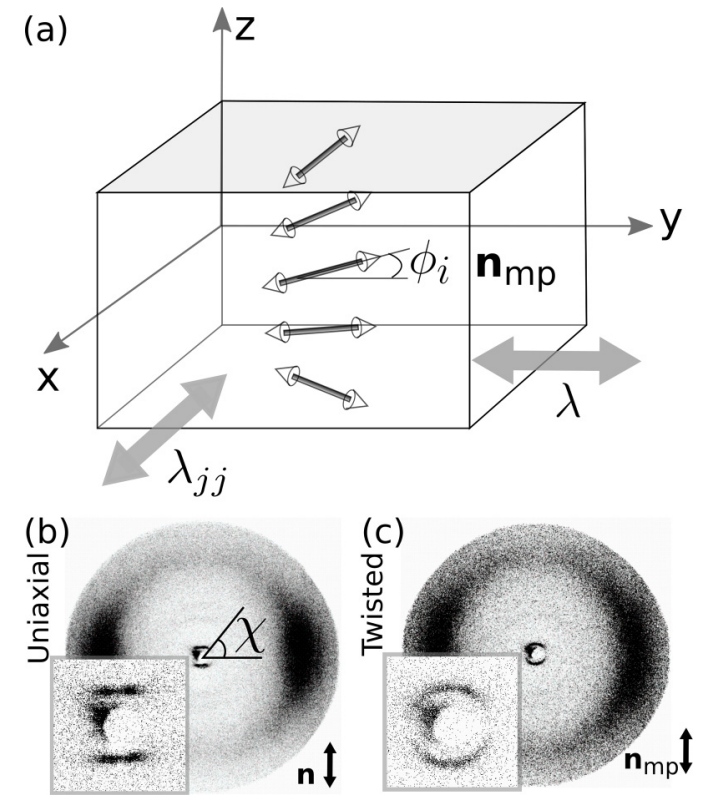

FIG. 1. (a) Model of a twisted nematic liquid crystal elastomer with the helix axis along $z$ and the initial midplane director $\mathbf{n}_{\mathrm{mp}}$ at an angle $\phi_{i}$ to the applied stretch $\lambda$. (b) Diffractogram of a uniaxial (no twist) liquid crystal elastomer film used to generate an azimuthal intensity profile $I(\chi)$ and model the twisted and stretched twisted nematic elastomers. Inset: zoom of small-angle scattering from smectic clusters. (c) The diffractogram of a twisted nematic liquid crystal elastomer with no stretch and, in the inset, the zoom of small-angle scattering reveal broadened reflexes due to the twisted configuration. The effect of an off-center beam stop is also visible in both inset images.

shift in the helix phase $\phi_{i}$, given by the director rotation at the film midplane $\mathbf{n}_{\mathrm{mp}}$ as depicted in Fig. 1(a).

The Warner untwisting model was previously experimentally tested with side-chain polysiloxane-based shortpitch cholesteric LCEs [17]. In the present work, the twisted nematic LCE samples were prepared using the same click-chemistry and oligomer preparation as described in [3]. Briefly, we synthesized an oligomer prior to LCE film fabrication by a thiol-acrylate click reaction; the reactive liquid crystal monomer, 1,4-bis-[4-(6-acryloyloxyhexyloxy)benzoyloxy]-2-methylbenzene (RM82), was chain extended by Michael addition with 1,5-pentanedithiol (PDT). In a typical synthesis, we mixed $12.5 \mathrm{~g}$ of RM82 with $5.06 \mathrm{~g}$ of 1,5-PDT in $120 \mathrm{~mL}$ of dichloromethane (DCM) with three drops of base catalyst. After $16 \mathrm{~h}$ of stirring at room temperature, we rinsed the solution in a separation funnel with $1 M$ hydrochloric acid $(\mathrm{HCl}), 0.1 M \mathrm{HCl}$, and deionized water successively. We dried the DCM-product mixture with $25 \mathrm{~g}$ of magnesium sulfate for $30 \mathrm{~min}$ and then poured it through a filter. We added $50 \mathrm{mg}$ of cross-linking inhibitor, butylated hydroxytoluene, to the clear DCM and product mixture prior to rotary evaporation and direct vacuum until a thick white oligomer remained. We found the oligomer could be stored at $-30{ }^{\circ} \mathrm{C}$ for up to 2 months. Prior to fabrication of LCE films, we melted and mixed into the oligomer additional RM82 with photoinitiator to create an LCE precursor melt that could then be used to form cross-linked films.

To program the twisted monodomain, we poured the LCE precursor melt onto a glass substrate coated with a uniaxially programmed layer of the photoalignment dye, Brilliant Yellow. We then sandwiched the melt with another photoprogrammed glass substrate such that the programmed uniaxial directions of the two substrates were aligned at $80^{\circ}$ to each other. This angle, less than $90^{\circ}$, was chosen to ensure that a monodomain of single twist handedness was achieved throughout the sample with no defects. We set the thickness of the LCE by polyimide tape placed on one substrate, which resulted in approximately 70 - $\mu$ m-thick LCE films as measured by confocal laser profilometry. After cooling the oligomer and photocuring to form a cross-linked elastomer, we separated the glass substrates by submerging them in water to obtain a freestanding film of twisted nematic LCE for testing. We obtained individual test pieces of a 20 by $10 \mathrm{~mm}^{2}$ LCE film with varying $\phi_{i}$ between the long axis and $\mathbf{n}_{\mathrm{mp}}$ by laser cutting them from the larger monodomain samples. We stretched test pieces along their long axis and perpendicular to the incident $\mathrm{x}$-ray beam in a custom-built tensile tester. See the Supplemental Material for more details on the experimental procedures [18].

We collected x-ray diffractograms of an exemplary unstretched uniaxial nematic film [Fig. 1(b)] and unstretched twisted nematic film [Fig. 1(c)]. Diffuse peaks inset in Figs. 1(b) and 1(c) indicate the presence of short-range smectic clustering. The twist structure is clearly seen by broadening of both the wide-angle and small-angle scattering reflexes. We used the azimuthal wide-angle profile of the unstretched uniaxial nematic $x$-ray diffractogram to model the azimuthal profile of the twisted nematic films by summing through the film thickness from thin uniaxial slices with orientations sampled from the twisted nematic director $\phi(z ; \lambda)$ profile. The constitutive equation of the adapted model giving the $\phi$ profile is

$$
\tan (2 \phi)=\frac{2 \lambda \lambda_{j j}(r-1) \sin \left(2 \tilde{q} z+2 \phi_{i}\right)}{(r-1)\left(\lambda^{2}+\lambda_{j j}^{2}\right) \cos \left(2 \tilde{q} z+2 \phi_{i}\right)+(r+1)\left(\lambda^{2}-\lambda_{j j}^{2}\right)} .
$$

This equation describes how the stretch $\lambda$ causes the director to rotate nonuniformly and the helix pitch to change through its thickness; the new wave number is $\tilde{q}=q_{0} / \lambda_{z z}$, where $\lambda_{z z}=\lambda^{-\beta}$ and $\lambda_{j j}=\lambda^{-1+\beta}$ and thus $\lambda_{z z} \lambda_{j j} \lambda=1$. Here, we have used $\lambda_{j j}$ to specifically indicate the direction normal to both the helix and the applied stretch $\lambda$, which we vary in both direction and magnitude. For a short-pitch cholesteric LCE, $\beta$ was found to be exactly $2 / 7[10,19,20]$. In a twisted nematic LCE, $\beta$ will generally depend on $\phi_{i}$ and vary with the applied stretch $\lambda$. However, for the purposes of qualitatively modeling 


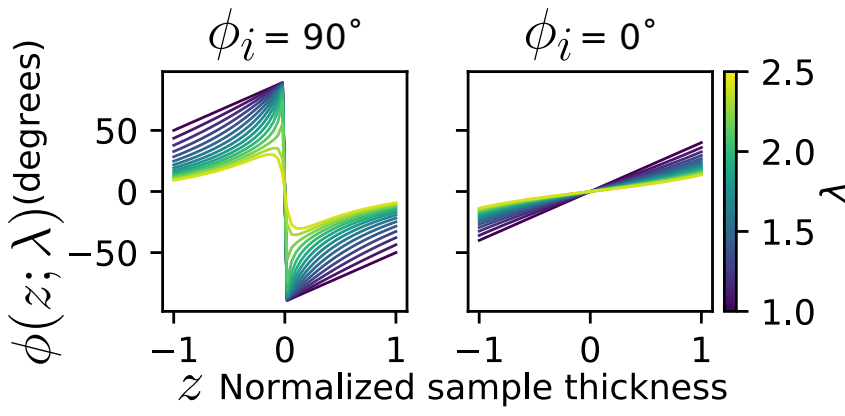

FIG. 2. Adapted model for the helical director profile as a function of the applied stretch $\lambda$ when the midplane director is initially perpendicular to the stretching axis (left) and when the midplane director is initially parallel to the stretching axis (right).

data in our experiments we find good approximation by fixing $\beta=2 / 7$ for all stretches and initial orientations of the film relative to the applied stretch. As in the original model, we also consider the polymer network anisotropy $r$, "the ratio of the effective step lengths parallel and perpendicular to the director" [10]. Examples of the modeled untwisting director profile for stretch perpendicular and parallel to $\mathbf{n}_{\mathrm{mp}}$ as a function of stretch are presented in Fig. 2. The wide-angle azimuthal intensity from the uniaxial diffractogram $I_{\mathrm{U}}(\chi)$ was then shifted by $\phi$ determined from the untwisting director model and integrated over many slices in $z$ through the thickness to calculate a predicted form of the twisted structure azimuthal intensity: $I(\chi ; \lambda)=\int_{-1}^{1} I_{\mathrm{U}}[\chi+\phi(z ; \lambda)] d z$.

The wide-angle $(\chi)$ azimuthal intensity profiles $I(\chi ; \lambda)$ of quasistatically stretched twisted nematic LCE films are presented in Fig. 3 along with the respective fit model for each given $\phi_{i}$. The models were fit by first tuning the $\phi_{i}$ parameter and then minimizing fit error over the chain anisotropy parameter $r$ for the fixed $\phi_{i}=75^{\circ}$. We found the chain anisotropy to be $r \approx 8.5$, which is somewhat larger than in side-chain LCEs [10] but is in line with measurements of other main-chain liquid crystal polymers [21] and implies a critical stretch $\lambda_{c}=r^{2 / 7} \approx 1.85$. As noted above, this relationship between $r$ and $\lambda_{c}$ was derived for short-pitch cholesteric elastomers. Regardless, the important qualitative features of the observed azimuthal intensity scattering profiles and the modeled profiles match well: a critical transition appeared when we stretched the twisted nematic LCE film such that a component of the helix pitch is perpendicular to the stretching direction (Fig. 3, $\phi_{i}=75^{\circ}$ ). In this case, the director helix initially increases in twist (decreased pitch length) so that the azimuthal x-ray signal becomes flatter. As stretching continues, the director bunches up along the pinned director perpendicular to the stretching direction, $\chi=1$, until a critical stretch. After the critical stretch, the director helix begins to unwind, and the signal has the appearance of uniaxial LCE. Additionally, we observed only increased order as we stretched the twisted nematic LCE film such that a component of the helix director was along the stretching direction.

We also laser cut test strips, $20 \mathrm{~mm}$ by $4 \mathrm{~mm}$ by $70 \mu \mathrm{m}$, for tensile mechanical tests from a sample and stretched them at a constant rate, as shown in Fig. 4(a). For increasing $\phi_{i}$, the modulus for small stretch, $\lambda<1.05$, monotonically increased

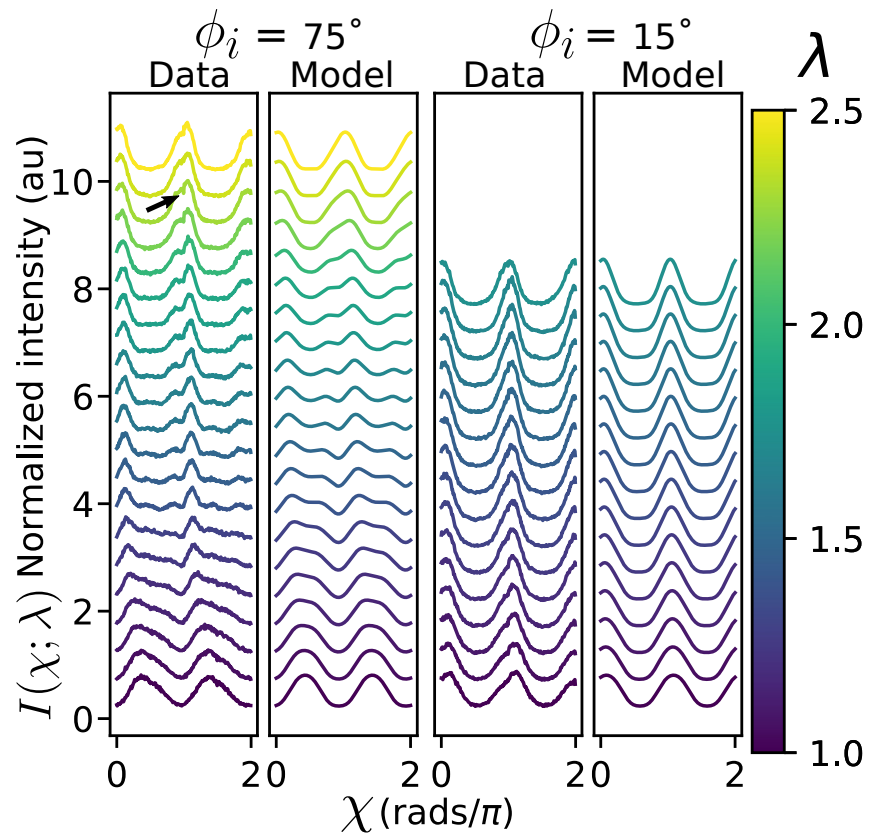

FIG. 3. Azimuthal x-ray data and comparison to the model for twisted nematic LCE films stretched $\phi_{i}=75^{\circ}$ to the midplane director $\mathbf{n}_{\mathrm{mp}}$ and $15^{\circ}$ to $\mathbf{n}_{\mathrm{mp}}$. Each intensity $I(\chi)$ curve is normalized to its own integral and offset a constant amount for visibility. Samples were quasistatically stretched to breaking. The arrow indicates the apparent presence of the pinned director at $\lambda=2.3$.

from 2.5 to $18 \mathrm{MPa}$. However, the stretch at break was nearly constant, $\lambda \approx 1.9$ for all cases where the stretch was applied such that an initial portion of the helical director distribution was along the stretching direction, i.e., $\phi_{i}<45^{\circ}$. At $\phi_{i}=45^{\circ}$, the stretch at break was also similar, which we attribute to a possible alignment error of the LCE film during laser cutting of the test strips or fixing in the tensile tester. For $\phi_{i}>45^{\circ}$, the stretch at break begins to grow, and the soft-mode elastic response of the LCE director realigning with stretch is apparent from the regions of constant induced stress. In uniaxial LCEs, the stretch at break tends to grow with increasing angle between alignment and stretching directions [22].

When we stretched samples between crossed polarizers, we observed different behaviors in the transmitted light intensity dependent on $\phi_{i}$ [Fig. 4(b)]. For both $\phi_{i}=0^{\circ}$ and $\phi_{i}=90^{\circ}$, the director guides the transmitted light polarization such that both films appear initially bright. As the films stretched, the film with $\phi_{i}=90^{\circ}$ became darker much faster and more completely before breaking than the film with $\phi_{i}=$ $0^{\circ}$, which broke before the transmittance reached half its initial relative intensity. For both films, because the director at the surface where light entered the film was not exactly in line with either of the polarizers, the light initially became elliptically polarized so that it was guided to partially pass the analyzer. During stretching, the director in the film with $\phi_{i}=0^{\circ}$ never reached alignment with the analyzer axis before breaking. However, as the $\phi_{i}=90^{\circ}$ film stretch increased, both the top and bottom directors became closer to perpendicular, with the polarizer axis thus guiding the light polarization into extinction by the analyzer (i.e., in the Mauguin regime). 
(a)

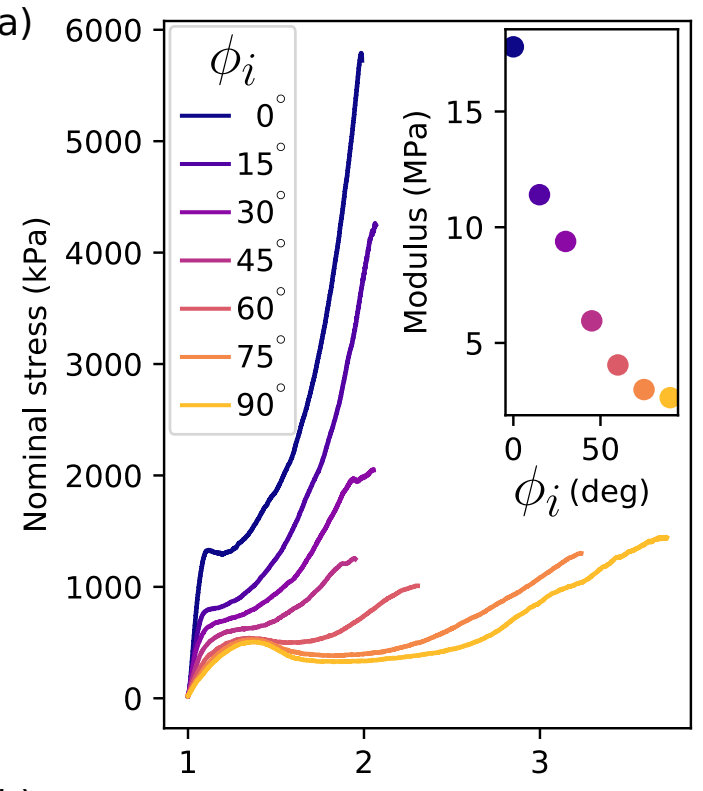

(b)

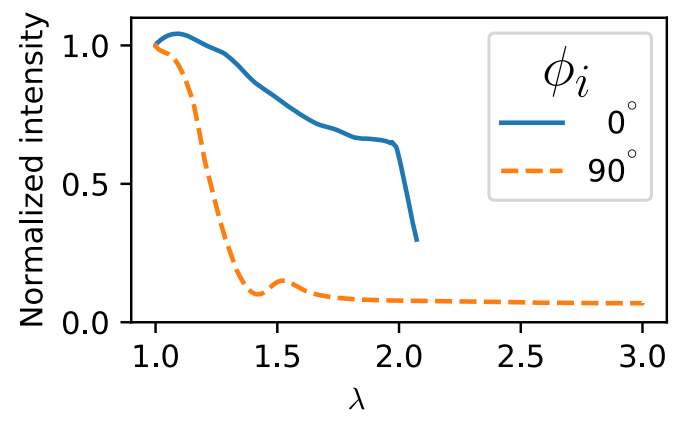

FIG. 4. (a) Mechanical and (b) cross-polarized optical stretch response anisotropy. In the inset in (a), the modulus for the first 0.05 of stretch changed by nearly an order of magnitude. All samples were stretched to breaking at $0.02 \mathrm{~s}^{-1}$.

We also observed a slight uptick in the transmitted light of the $\phi_{i}=90^{\circ}$ film near $\lambda \approx 1.5$ which we believe is due to a particular matching of the director, birefringence, and incident polarization to create an elliptical polarization which is not as well extinguished.

In the configuration used in the x-ray experiments here, we did not resolve in detail the smecticlike reflexes. However, it is clear that there is a smectic structure in the LCE films that we tested. These structures have also appeared in a few other similar systems [1,2]. The higher-order smectic structure likely explains the large elastic anisotropy seen here in mechanical tests [Fig. 4(a)] as purely nematic LCEs do not typically exhibit such large anisotropy [23]. A further signature of the smectic- $C$ structure can be seen in the stretched film with $\phi_{i}=0^{\circ}$, where we observed a brief plateau in the induced stress, which has been explained by sliding along planes of the smectic- $C$ phase [13]. Unlike previous results examining smectic- $A$ elastomers [24-26], the present results appear to fit a model of the smectic layer normal rotating in the plane of the stretch and not forming a cone of chevroned layers. This is consistent with the lower point symmetry in smectic- $C$ elastomers and their ability to deform softly.

However, a monodomain of the smectic- $C$ elastomer through the entirety of the film is not possible due to the programmed twisted configuration. Theory predicts an incompatibility between twisted and smectic structures, and for short twisting pitch, this results in the so-called twist-grain boundary. In this work, we suspect that the phase structure is actually similar to a nematic cybotactic phase, that is, clusters of short-range smectic ordering with nematic lubrication between clusters that permits an overall twisted structure with no defects, such as twist-grain boundaries in the smectic phase. In other words, it appears that the smectic and nematic phases are both present, but twisting occurs only in the nematic phase. Similar observations of smectic clustering in a twisted nematic have been made in thermotropic small-molecule liquid crystals [27]. We suspect that the smectic clusters arise from pretransitional fluctuations which have been trapped by cross-linking the polymer network.

We have prepared and characterized an elastomer with both twisted nematic and smectic- $C$ phases, which combines mechanical and optical features of both phases in a single material. The ability to structure both director field twist and bend deformations as well as large mechanical anisotropy plays a crucial role in many developing applications of LCEs. Our results indicate that tailoring the oligomers and director field structure used in making LCE devices will play an important role in the trade-off between these features, which are important, for example, in electroactive LCE actuators and sensors [28]. Future studies will also probe how short-range smectic clusters grow and are trapped by cross-linking the elastomer network. Furthermore, the mechanical model can be developed to find the dependence of $\beta$ on $\phi_{i}$ and the total initial twist $[11,19]$. Together, these studies will unveil a more complete picture of the connection between the microscopic structure and macroscopic mechanical properties.

We thank J. Lagerwall and M. Warner for helpful discussions. Z.S.D. was supported by the Alexander von Humboldt Foundation for this work. Z.S.D., J.F., and M.S. also acknowledge support from the Max-Planck-Gesellschaft.
[1] M. O. Saed, R. H. Volpe, N. A. Traugutt, R. Visvanathan, N. A. Clark, and C. M. Yakacki, High strain actuation liquid crystal elastomers via modulation of mesophase structure, Soft Matter 13, 7537 (2017).

[2] N. P. Godman, B. A. Kowalski, A. D. Auguste, H. Koerner, and T. J. White, Synthesis of elastomeric liquid crystalline polymer networks via chain transfer, ACS Macro Lett. 6, 1290 (2017).
[3] Y. Xia, X. Zhang, and S. Yang, Instant locking of molecular ordering in liquid crystal elastomers by oxygen-mediated thiol-acrylate click reactions, Angew. Chem., Int. Ed. 57, 5665 (2018).

[4] O. Yaroshchuk and Y. Reznikov, Photoalignment of liquid crystals: Basics and current trends, J. Mater. Chem. 22, 286 (2011). 
[5] T. H. Ware, M. E. McConney, J. J. Wie, V. P. Tondiglia, and T. J. White, Voxelated liquid crystal elastomers, Science 347, 982 (2015).

[6] W. Feng, D. J. Broer, and D. Liu, Oscillating chiral-nematic fingerprints wipe away dust, Adv. Mater. 30, 1704970 (2018).

[7] H. Finkelmann, S. T. Kim, A. Muñoz, P. Palffy-Muhoray, and B. Taheri, Tunable mirrorless lasing in cholesteric liquid crystalline elastomers, Adv. Mater. 13, 1069 (2001).

[8] V. P. Tondiglia, M. Rumi, I. U. Idehenre, K. M. Lee, J. F. Binzer, P. P. Banerjee, D. R. Evans, M. E. McConney, T. J. Bunning, and T. J. White, Electrical control of unpolarized reflectivity in polymer-stabilized cholesteric liquid crystals at oblique incidence, Adv. Opt. Mater. 6, 1800957 (2018).

[9] R. Kizhakidathazhath, Y. Geng, V. S. R. Jampani, C. Charni, A. Sharma, and J. P. F. Lagerwall, Facile anisotropic deswelling method for realizing large-area cholesteric liquid crystal elastomers with uniform structural color and broad-range mechanochromic response, Adv. Funct. Mater. 30, 1909537 (2020).

[10] M. Warner, E. M. Terentjev, R. B. Meyer, and Y. Mao, Untwisting of a Cholesteric Elastomer by a Mechanical Field, Phys. Rev. Lett. 85, 2320 (2000).

[11] Y. Mao, E. M. Terentjev, and M. Warner, Cholesteric elastomers: Deformable photonic solids, Phys. Rev. E 64, 041803 (2001).

[12] Y. Sawa, F. Ye, K. Urayama, T. Takigawa, V. Gimenez-Pinto, R. L. B. Selinger, and J. V. Selinger, Shape selection of twistnematic-elastomer ribbons, Proc. Natl. Acad. Sci. USA 108, 6364 (2011).

[13] J. M. Adams and M. Warner, Mechanical response of smectic- $C$ elastomers, Phys. Rev. E 77, 021702 (2008).

[14] J. W. Goodby, Twist grain boundary and frustrated liquid crystal phases, Curr. Opin. Colloid Interface Sci. 7, 326 (2002).

[15] A. D. Vries, X-ray photographic studies of liquid crystals I. A cybotactic nematic phase, Mol. Cryst. Liq. Cryst. (1969-1991) 10, 219 (1970).

[16] C. Zhang, M. Gao, N. Diorio, W. Weissflog, U. Baumeister, S. Sprunt, J. T. Gleeson, and A. Jákli, Direct Observation of
Smectic Layers in Thermotropic Liquid Crystals, Phys. Rev. Lett. 109, 107802 (2012).

[17] P. Cicuta, A. R. Tajbakhsh, and E. M. Terentjev, Evolution of photonic structure on deformation of cholesteric elastomers, Phys. Rev. E 65, 051704 (2002).

[18] See Supplemental Material at http://link.aps.org/supplemental/ 10.1103/PhysRevMaterials.4.105601 for details of the experimental methods.

[19] P. A. Bermel and M. Warner, Photonic band structure of highly deformable self-assembling systems, Phys. Rev. E 65, 010702(R) (2001).

[20] P. A. Bermel and M. Warner, Photonic band structure of cholesteric elastomers, Phys. Rev. E 65, 056614 (2002).

[21] J. F. D'Allest, P. Maïssa, A. ten Bosch, P. Sixou, A. Blumstein, R. Blumstein, J. Teixeira, and L. Noirez, Experimental Evidence of Chain Extension at the Transition Temperature of a Nematic Polymer, Phys. Rev. Lett. 61, 2562 (1988).

[22] T. H. Ware, J. S. Biggins, A. F. Shick, M. Warner, and T. J. White, Localized soft elasticity in liquid crystal elastomers, Nat. Commun. 7, 10781 (2016).

[23] M. Warner and E. M. Terentjev, Liquid Crystal Elastomers (Oxford University Press, Oxford, 2007).

[24] J. M. Adams and M. Warner, Elasticity of smectic- $A$ elastomers, Phys. Rev. E 71, 021708 (2005).

[25] O. Stenull, T. C. Lubensky, J. M. Adams, and M. Warner, Smectic- $C$ tilt under shear in smectic- $A$ elastomers, Phys. Rev. E 78, 021705 (2008).

[26] C. M. Spillmann, J. H. Konnert, J. M. Adams, J. R. Deschamps, J. Naciri, and B. R. Ratna, Strain analysis of a chiral smectic- $A$ elastomer, Phys. Rev. E 82, 031705 (2010).

[27] L. Z. Ruan, M. A. Osipov, and J. R. Sambles, Coexisting Nematic and Smectic- $A$ Phases in a Twisted Liqui-Crystal Cell, Phys. Rev. Lett. 86, 4548 (2001).

[28] Z. S. Davidson, H. Shahsavan, A. Aghakhani, Y. Guo, L. Hines, Y. Xia, S. Yang, and M. Sitti, Monolithic shapeprogrammable dielectric liquid crystal elastomer actuators, Sci. Adv. 5, eaay0855 (2019). 